\title{
Insights into the molecular mechanism of RGL2-mediated inhibition of seed germination in Arabidopsis thaliana
}

\author{
Petra Stamm¹, Pratibha Ravindran ${ }^{1}$, Bijayalaxmi Mohanty ${ }^{2}$, Ee Ling Tan ${ }^{1}$, Hao Yu ${ }^{1,3}$ and Prakash P Kumar ${ }^{1,3^{*}}$
}

\begin{abstract}
Background: Seed germination is of immense significance for agriculture and has been studied for centuries. Yet, our understanding of the molecular mechanisms underlying regulation of dormancy and germination is still in its infancy. Gibberellins are the key phytohormones that promote germination, and the DELLA protein RGL2 is the main signalling intermediate involved in this response. Germination is completely inhibited if functional RGL2 is overexpressed and/or stabilized; however, the molecular mechanisms of RGL2 function are still largely unknown. We therefore attempted to shed light onto some of the genetic events downstream of RGL2.
\end{abstract}

Results: Gene ontology of the transcriptome differentially regulated by RGL2, as well as extensive cross-comparison with other available microarray data indicates that RGL2-mediated inhibition of germination causes seeds to enter a state of dormancy. RGL2 also appears to differentially regulate a number of transcription factors, many of which are known to be involved in light- or phytohormone-mediated aspects of germination. A promoter analysis of differentially expressed genes identified an enrichment of several motifs that can be bound by specific transcription factors, for example GAMYB, ARF1, or Dof-type zinc fingers. We show that Dof-binding motifs indeed play a role in RGL2-mediated transcription. Using Chromatin Immunoprecipitation (ChIP), we show that RGL2 directly downregulates at least one cell wall modifying enzyme, which is predicted to constrain cell growth thereby leading to inhibition of seed germination.

Conclusions: Our results reveal that RGL2 controls various aspects of germination. Through the repression of cell wall modifying enzymes, cell growth is directly constrained to inhibit germination. Furthermore, RGL2 likely interacts with various types of proteins to regulate transcription, and differentially regulates several transcription factors. Collectively, our data indicate that gibberellins, acting via RGL2, control several aspects of seed germination.

Keywords: RGL2, Seed germination, Dormancy, Gibberellins, DELLAs, Arabidopsis microarray

\section{Background}

Gibberellins are phytohormones regulating growth and development throughout a plant's life cycle; they are essential in processes such as stem elongation, floral development and seed germination [1-4]. The importance of gibberellins in these processes is most obvious in gibberellin-deficient mutants; GIBBERELLIC ACID REQUIRING 1 (GA1) encodes for the ent-kaurene synthetase A of the gibberellin

\footnotetext{
* Correspondence: dbskumar@nus.edu.sg

'Department of Biological Sciences, Faculty of Science, National University of Singapore, Singapore 117543 , Singapore

${ }^{3}$ Temasek Life Sciences Laboratory, National University of Singapore, 1

Research Link, Singapore 117604, Singapore

Full list of author information is available at the end of the article
}

biosynthetic pathway, ga1 plants are therefore unable to synthesise gibberellins. These mutants are extremely dwarfed, male-sterile, and most importantly their seeds fail to germinate without exogenous gibberellin $[5,6]$.

Significant progress has been made in recent years in elucidating the gibberellin signalling pathway $[7,8]$. The gibberellin signal is perceived by the soluble receptors GIBBERELLIN INSENSITIVE DWARF 1 (OsGID1 or OsGID1-like) $[9,10]$. Arabidopsis contains three GID1-like genes, GID1a, GID1b and GID1c [11]. The gibberellinGID1 interaction triggers the degradation of DELLA proteins, the major negative regulators of gibberellin signalling, via the $26 \mathrm{~S}$ proteasome pathway [12-15]. The 
gibberellin-specific F-box proteins OsGID2 and SLEEPY1 (AtSLY1) mediate this degradation [16-19].

DELLA proteins are a subfamily of the GRAS family of transcriptional regulators [20], named after their highly conserved N-terminal DELLA motif, which mediates gibberellin-responsiveness [21-23]. In Arabidopsis, there are five DELLA proteins: GIBBERELLIN INSENSITIVE (GAI), REPRESSOR OF ga1-3 (RGA), RGA-like1 (RGL1), RGL2 and RGL3 [13,24-26]. Extensive genetic studies using various combinations of DELLA knock-out mutations have elucidated overlapping as well as distinct functions of each protein in repressing plant growth and development: RGA and GAI are the main repressors of stem elongation, whereas floral development is regulated by a combination of RGA, RGL1 and RGL2, and RGL2 is the key DELLA protein repressing seed germination [24,27-31].

However, the events downstream of DELLAs in the gibberellin-mediated regulation of growth and development are less well understood. Until recently, it was not clear how DELLA proteins repress gibberellin-mediated gene expression. Although they have been classified as transcriptional regulators, they do not have conserved DNA binding domains. A major break-through in understanding the molecular mechanism of DELLAaction was achieved when the direct interaction of DELLA proteins with PHYTOCHROME INTERACTING FACTOR 3 (PIF3) and PIF4 was shown [32,33]. Since then, the main mode of DELLAs in regulating transcription is thought to occur via the sequestering of transcription factors. Recently, the interaction in yeast with more members of the basic helix-loop-helix (bHLH) subfamily 15, namely PIF3-like 5 (PIL5), PIL2 and SPATULA (SPT) was shown; the authors thus hypothesised that DELLAs could interact with all members of this subfamily [34]. This further corroborates the conclusion that the main molecular mechanism of DELLA function is their interaction with transcription factors, which leads to the formation of inactive complexes [35]. This model has been further revised to show that DELLA proteins are also able to activate transcription by sequestration of inhibitors [36-38].

Here, we focus on gibberellin-mediated regulation of germination, in particular the molecular mechanism by which the DELLA protein RGL2 suppresses germination. Germination is a complex process of three phases, each being tightly regulated at various levels. Phase I describes the intake of water, during which the seed imbibes, whereas in phase II metabolic processes are reinitiated (also called germination sensu stricto), and in phase III the radicle emerges $[39,40]$. Seed germination is regulated by the balance of the two phytohormones abscisic acid and gibberellins, which inhibit and promote germination, respectively. Gibberellins function in late phase II of germination [41], a phase which is physiologically characterised mainly by post-imbibitional cell elongation in embryonic radicle and hypocotyl as well as endosperm weakening [42]. Gibberellins are therefore key players in the actual commitment of seeds to germination.

RGL2 is the major DELLA protein involved in repressing germination [25,31]. It performs this function, at least partly, through increasing abscisic acid biosynthesis as well as activities of ABI5 and ABI3 [43,44]. This has been further elucidated by Lee et al. [45], showing that RGL2 regulates abscisic acid release in the endosperm to control embryo growth. In fact, RGL2 has been identified as one of the genes to be involved in the regulation of seed germination at the phase II to phase III transition [46]. Despite all these findings, it remains unclear exactly how RGL2 suppresses this complex process of germination. Therefore, elucidation of the molecular mechanism of RGL2 function would not only allow us to gain a deeper understanding of gibberellin-mediated germination, but also enable us to manipulate details of germination, for example to prevent pre-harvest sprouting in crops, at the same time ensure full and synchronous germination upon sowing.

In this study, we show that RGL2 function causes seeds to enter a state of dormancy. Microarray analysis showed that RGL2 up-regulates several genes associated with dormant states of seeds. Enforcing dormancy is partly achieved by RGL2 directly inhibiting transcription of cell wall-modulating genes ALPHA EXPANSIN3 (EXPA3) and EXPA8, by binding to their promoters, perhaps as a complex with as yet unidentified transcription factors. Thus, RGL2 directly affects cell growth. These data suggest that RGL2 inhibits seed germination both directly and indirectly.

\section{Results}

RGL2-mediated transcriptome in non-germinating seeds

In order to identify genes that are differentially regulated by RGL2 to repress germination, an oligonucleotidebased DNA microarray analysis (Agilent 60-mer gene expression microarray, $4 \mathrm{x} 44 \mathrm{k}$ ) was performed. We compared the transcriptome of the gal-3 rga-t2 mutant seeds, which are unable to germinate, with that from ga1-3 rga-t2 rgl2-1, in which germination is rescued to near wild type levels (Figure 1). Our aim was to identify target genes specifically regulated by RGL2. We therefore chose seeds from both gibberellin-deficient as well as RGA knock-out backgrounds. With the gibberellindeficient background we aimed to exclude gibberellinmediated, DELLA-independent target genes [47]. The knock-out of $R G A$ in both genotypes was meant to further narrow our results down to RGL2-specific targets, since RGA plays a minor, additive role in repressing seed germination [31]. RNA was obtained from seeds 

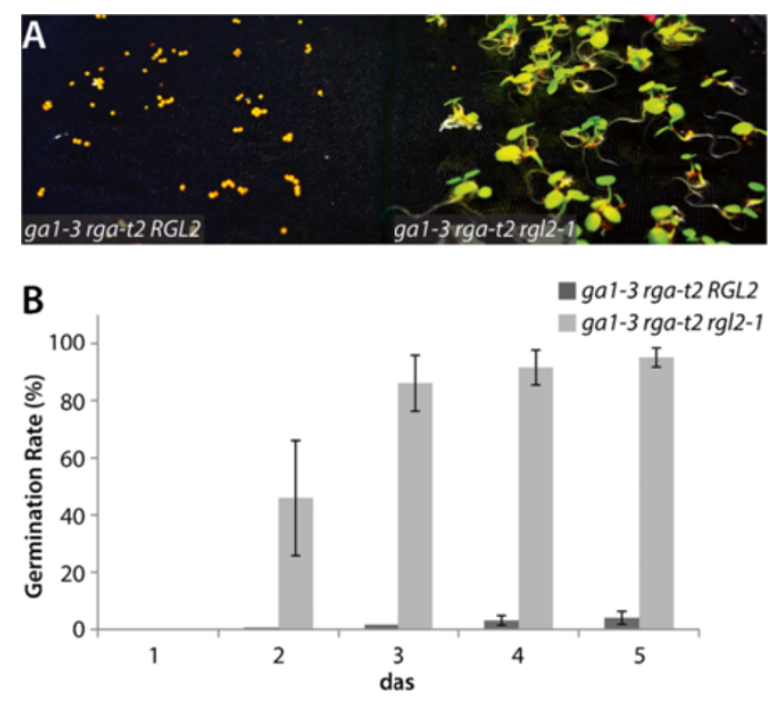

Figure 1 A mutation in RGL2 can rescue the non-germinating phenotype of the gibberellin-deficient mutant. Seeds of the double mutant ga1-3 rga-t2 fail to germinate, due to stabilised RGL2, whereas the mutation of RGL2 in the triple mutant gal-3 rga-t2 rgl2-1 rescues germination to near wild-type levels. Germination rate was determined over a period of five days following stratification of seeds at $4^{\circ} \mathrm{C}$ for five days. Error bars indicated SD of three biological replicates. Seeds were deemed germinated when the radicle had visibly emerged from the seed coat. Pictures are representative of seeds/seedlings five days after imbibition.

after imbibition for five days at $4^{\circ} \mathrm{C}$. This cold treatment promotes as well as synchronises germination [41,48]. Hence, the RNA isolated from seeds treated in such a way should represent the steady-state transcriptomes of seeds poised for germination. Two microarray replicates were performed for each genotype with independently obtained RNA. We applied stringent analysis criteria, referring to genes as being RGL2-up-regulated (RGL2UP) or RGL2-down-regulated (RGL2-DOWN), only if in both hybridisations the signal ratio of ga1-3 rga-t2 to ga1-3 rga-t2 rgl2-1 was equal to or more than 2-fold different, with a p-value cut-off at 0.01 . Using these criteria, we identified 607 genes as putative RGL2-regulated genes, 253 being up-regulated (RGL2-UP) and 355 being down-regulated (RGL2-DOWN) (Additional file 1).

To further understand events during the RGL2mediated arrest in germination, and to eliminate secondary effects of the germination arrest, we classified our gene lists by gene ontology (GO) (http://www.arabidopsis. org/tools/bulk/go/index.jsp and http://bioinfo.cau.edu.cn/ agriGO/index.php) (Additional file 2). In our data, 350 and 249 of down- and up-regulated genes, respectively, were annotated. As expected, transcripts related to postembryonic morphogenesis were significantly $(p<0.01)$ enriched in RGL2-DOWN. In accordance with that, GO terms related to cellular components 'plasma membrane' and 'plant-type cell wall' were significantly enriched. We further found a significant over-representation of the molecular function terms 'hydrolase activity', 'carboxylesterase activity' $(\mathrm{p}<0.05)$ and 'serine-type carboxypeptidase activity' $(\mathrm{p}<0.01)$. The predominant functional themes in RGL2-UP were related to responses to various exogenous and endogenous cues; GO terms 'response to oxidative stress,' 'response to abiotic stimulus,' 'toxin catabolic processes' and 'response to gibberellin' were significantly enriched $(\mathrm{p}<0.01)$. With respect to molecular functions, we found a significant $(\mathrm{p}<0.01)$ enrichment of genes assigned 'glutathione transferase activity' and 'UDPglucosyl transferase activity'. Furthermore, 33 genes (13\%) were found in the 'transcription factor activity' category. These include several uncharacterised transcription factors containing MYB, basic helix-loop-helix (bHLH) or AP2 domains, as well as the Homeodomain-Leucinezipper (HD-Zip) genes ATHB4 and ATHB7, MYB111, WUSCHEL-related homeobox 2 (WOX2) and WRKY40, among others. Furthermore, several hormone pathways seem to be affected; genes involved in the response to salicylic acid and gibberellins are significantly enriched in the up-regulated genes, whereas the response to auxin appears as an enriched term in down-regulated genes. Also, many of the up-regulated transcription factors appear to be either responsive to or modulate responses to the phytohormones abscisic acid, ethylene, salicylic acid, brassinosteroids and gibberellins.

\section{RGL2-mediated transcriptome overlaps with transcriptomes of dormant wild-type seeds}

Since germination is a complex process mediated by a vast number of cues, a large number of differentially regulated genes likely represent secondary responses due to the arrest of germination, rather than a direct effect of RGL2 function. We therefore aimed to narrow down our gene lists by performing cross-comparisons with other available microarray data related to seeds, germination, gibberellins and DELLA proteins. One data set, generated by Cao et al. [47], contains gene expression profiles of germinating seeds and developing flowers, respectively, of wild type cv. Landsberg erecta (Ler), ga13 , and the gal-3 rga gai rgl1 rgl2 quintuple mutant, identifying both DELLA-dependent as well as DELLAindependent or partially dependent genes. Another study identified direct target genes of RGA in seedling shoots [49]. We also compared our data with the list of differentially regulated genes in seeds of the comatose (cts-1) mutant, compared to dormant (D) and after-ripened (AR) seeds of wild type cv. Landsberg erecta (Ler) [50]. COMATOSE (CTS) encodes for a peroxisomal ATP binding cassette transporter, which is required for seedling establishment and survival just before radicle protrusion, downstream of RGL2; thus, cts-1 mutant seeds remain "forever dormant". Lastly, PHYTOCHROME 
INTERACTING FACTOR 3-LIKE5 (PIL5), encoding for a bHLH transcription factor, is a key negative regulator of germination [51], and directly interacts with DELLA proteins [34]. Therefore, we also compared our data set with direct target genes of PIL5 that were identified by both Chromatin Immunoprecipitation-chip (ChIP-chip) and microarray data [52].

Despite various differences between these data sets, most importantly the experimental set-up (Affymetrix ATH1 vs. Agilent $4 x 44 k$ array), we observed a considerable overlap of gene expression profiles (Additional file 3). Of our RGL2UP list, 89 genes (35\%) overlap with at least one of these data sets, 58 of which are up-regulated in dormant seeds as identified by Carrera et al. [50], whereas 146 genes are unique for our data. Likewise, 177 genes (50\%) of RGL2DOWN genes were identified in at least one other data set, 110 of which are down-regulated in dormant seeds [50], with 177 genes being unique for our experiment. This not only validates the quality of our data, but also indicates the physiological state in which seeds appear to be arrested due to RGL2 action. Twelve of the genes that have been identified as direct target genes of PIL5 [52] can also be found in our data (six genes in RGL2-UP, six in RGL2-DOWN). In total, we found 75 and 17 down- and up-regulated genes, respectively, that were identified as DELLA-dependent transcripts in seeds [47]. Interestingly, some of these (three genes in RGL2-UP, six genes in RGL2-DOWN) appear to be DELLA targets in both flower buds and seeds. Thus, these genes likely represent direct targets of RGL2. Lastly, three of the genes directly targeted by RGA in seedling shoots [49] can also be found in our data, including GIBBERELLIN 20-OXIDASE (GA20ox) and GIBBERELLIN INSENSITIVE DWARF1b (GID1b). We therefore determined the expression levels of some of the genes that are likely direct targets of RGL2 in imbibed seeds of ga1-3 rgat2 and ga1-3 rga-t2 rgl2-1 (Figure 2). The as yet uncharacterised gene At2g45210 of the SMALL AUXIN UPREGULATED RNA (SAUR)-family, which appears to be a DELLA target gene in both flowers and seeds, is expressed at much higher levels in imbibed seeds of the ga1-3 rga-t2 mutant, indicating that it could be up-regulated by RGL2. We also confirmed the expression of the PIL5 target gene ALPHA EXPANSIN 8 (AtEXPA8) and the related $A t E X P A 3$, as well as the DNA-binding with one finger (Dof)-type transcription factor Dof2.1, all of which are down-regulated in the presence of RGL2. The observed expression levels are consistent with our microarray data, which further supports the hypothesis that these genes could be directly regulated by RGL2.

\section{Several cis-elements are abundant in promoters of RGL2-regulated genes}

We further analysed our data, aiming to understand how transcriptional regulation by RGL2 is coordinated.

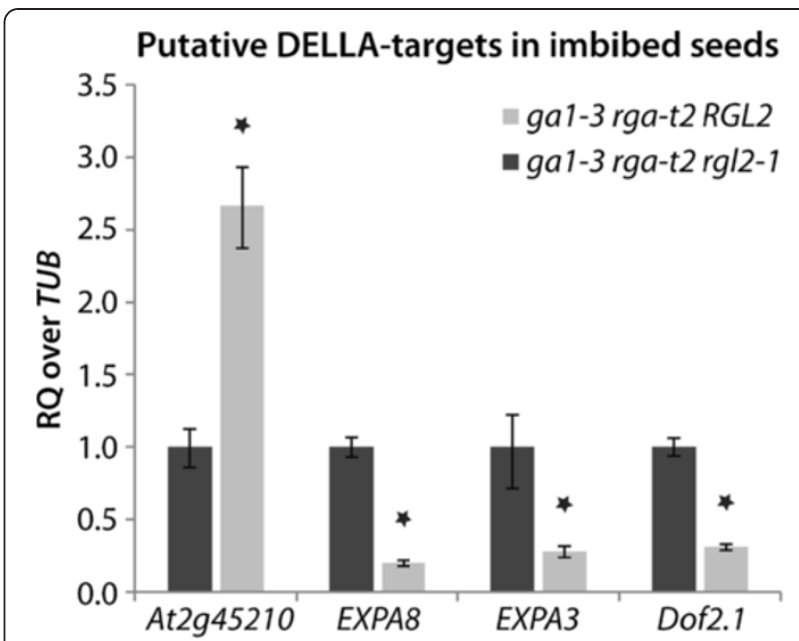

Figure 2 Relative expression levels of known DELLA target genes in seeds. Seeds were stratified in water, and RNA was extracted after five days. Expression levels of known DELLA-targets were determined by qRT-PCR in imbibed seeds of ga $-3 \mathrm{rga}$, relative to Tubulin (TUB), and compared to ga1-3 rga rg/2-1. Asterisks indicate a significant difference in expression levels $(p<0.05) . R Q=$ relative quantity of transcript.

For this, we identified motifs that are over-represented in the promoter regions of differentially regulated genes. Promoter regions $(-1,000$ to +200$)$ of the RGL2-regulated genes, were used for $a b$ initio detection of putative cis-elements using the Dragon Motif Builder algorithms. Motifs with occurrences of $20 \%$ or higher were matched with known promoter elements in the TRANSFAC, PLACE and AGRIS databases. This way, we identified 14 motifs in promoters of up-regulated (Table 1) and nine in promoters of down-regulated genes (Table 2). In promoters of both RGL2-UP and RGL2-DOWN genes, gibberellin response element (GARE)-like motifs, which are associated with GAMYBtype transcription factors, are the most significantly enriched motifs. Furthermore, motifs associated with the auxin signalling intermediate AUXIN RESPONSE FACTOR1 (ARF1) are significantly enriched in promoters of up-regulated genes, with three out of the 14 motifs detected belonging to this category. Interestingly, three out of the 14 motifs detected in RGL2-UP are associated with transcription factors of the Dof-type ('DNA-binding with one finger'), two of which (AAAAG element and TAAAG element) are present in more than $35 \%$ of the promoters analysed.

To further evaluate the biological significance of our $a b$ initio promoter motif prediction, we tested the Dofrecognised motifs for binding by the RGL2-complex in vivo. These motifs will henceforth be referred to as $\mathrm{M}_{\text {Dof }} 1$ (AAAAG element), $\mathrm{M}_{\text {Dof }} 2$ (TAAAG element) and $\mathrm{M}_{\text {Dof }} 3$ (AAGAA element). We generated reporter constructs containing three tandem copies of the consensus 
Table 1 Cis-elements enriched in promoters of RGL2-up-regulated genes

\begin{tabular}{|c|c|c|c|c|c|}
\hline $\begin{array}{l}\text { Consensus } \\
\text { sequence }\end{array}$ & $\begin{array}{l}\text { Putative } \\
\text { cis-element }\end{array}$ & $\begin{array}{l}\text { Associated type } \\
\text { of transcription } \\
\text { factor }\end{array}$ & $\%^{2}$ & $\mathrm{TIC}^{3}$ & e-value \\
\hline TTTTCAA & Pyrimidine box-like / PE1/AT-hook element-like / GT element-like & GAMYB / PF1, GT1 / GT3b & 46 & 14.53 & $2 e-005$ \\
\hline AAAAAAAAAG & AAAAG element / PE1/AT-hook element-like & Dof1, Dof4, Dof11, Dof16 / PF1 & 42 & 19.02 & $0 e+000$ \\
\hline AATAAAGA & TAAAG element & Dof4, Dof11, Dof16 & 37 & 14.50 & $0 e+000$ \\
\hline TCTCTCTाT & GAGA element-like / TCA-1 binding site & GAGA-binding factor BBR / TCA-1 & 32 & 17.00 & 2e-005 \\
\hline TATTTGTT & GARE-like / AuxRE-like & GAMYB / ARF1 & 31 & 16.08 & $0 \mathrm{e}+000$ \\
\hline TTAGTTT & Myb box-like & MYB1 & 29 & 15.03 & 2e-005 \\
\hline TTGTTTC & Pyrimidine box-like/GARE / GT element-like / AuxRE-like & GAMYB / HD PR2 / ARF1 & 27 & 16.23 & $0 e+000$ \\
\hline TTCTTG & TCA-1 binding site & TCA-1 (tobacco nuclear protein 1) & 26 & 16.00 & $0 \mathrm{e}+000$ \\
\hline TGCTTCTC & $\begin{array}{l}\text { CAMTA3 binding site / AuxRE-like / IDE1 } \\
\text { (iron-deficiency-responsive element 1) }\end{array}$ & CAMTA3 / ARF1 & 26 & 13.44 & $3 e-005$ \\
\hline AAAAAAATG & AT hook/PE1 element-like & PF1 / DBP & 23 & 16.45 & 2e-005 \\
\hline CTTATAT & TATA-box & TBP & 21 & 15.10 & $0 \mathrm{e}+000$ \\
\hline CAAGAATG & AAGAA motif & Dof2 & 20 & 14.03 & $0 \mathrm{e}+000$ \\
\hline GATTTGTT & GARE-like & GAMYB & 20 & 16.05 & $3 e-005$ \\
\hline AAGACAAA & GARE-like & GAMYB & 20 & 16.00 & $0 e+000$ \\
\hline
\end{tabular}

${ }^{1}$ Homology with motifs identified in Arabidopsis and other plant species (TRANSFAC, PLACE and AGRIS databases).

${ }^{2}$ Percent occurrence in critical promoters relative to background sequences.

${ }^{3}$ Total Information Content.

Promoter motifs identified in promoters of RGL2-UP, with consensus sequence, name and associated transcription factors.

sequence of each motif $5^{\prime}$ of the minimal 35 S promoter to drive the expression of GFP (Figure 3B) and transfected Arabidopsis mesophyll protoplasts derived from a 35S::RGL2-GR ga1-3 rga-t2 rgl2-1 transgenic line. These plants constitutively express $R G L 2$ fused to the rat glucocorticoid-receptor, which allows for inducible nuclear translocation by treatment with dexamethasone (DEX). This system was described as a 'potent tool in examining transcriptional activation' and has been successfully used before [30,53]. Without treatment, plants of this transgenic line bear no difference to those of the triple mutant ga1-3 rga-t2 rgl2-1. Upon DEX-treatment, RGL2-GR fusion protein can enter the nucleus, which in the intact plants reverts both flower development and seed germination to the gibberellin-deficient phenotype (to resemble the double mutant ga1-3 rga-t2), indicating that the fusion protein is functional (Figure 4). After transfection, protoplasts were either treated with (1) $0.01 \%$ ethanol (MOCK), (2) DEX in $0.01 \%$ ethanol, to induce RGL2 translocation into the nucleus, or (3) DEX plus gibberellic acid $\left(\mathrm{GA}_{3}\right)$, which will promote the degradation of RGL2. Thus, if the selected promoter

Table 2 Cis-elements enriched in promoters of RGL2-down-regulated genes

\begin{tabular}{|c|c|c|c|c|c|}
\hline $\begin{array}{l}\text { Consensus } \\
\text { sequence }\end{array}$ & $\begin{array}{l}\text { Putative of } \\
\text { cis-element }\end{array}$ & $\begin{array}{l}\text { Associated type } \\
\text { of transcription } \\
\text { factor }\end{array}$ & $\%^{2}$ & $\mathrm{TIC}^{3}$ & e-value \\
\hline TCCAAAAA & AT-hook/PE1 element-like / L-box/UV-B responsive element-like & PF1 & 38 & 14.52 & 2e-005 \\
\hline GTTाTाT & GARE-like / AT-hook/PE1 element-like & GAMYB / PF1 & 34 & 18.00 & $0 e+000$ \\
\hline TATAACAA & MYB box-like / GARE-like & GAMYB & 33 & 14.51 & $0 e+000$ \\
\hline TTТАTTТA & Box III-like & GT-1 & 32 & 17.13 & 2e-005 \\
\hline AAATTTCA & GT element-like & GT-1 & 30 & 15.01 & $0 e+000$ \\
\hline TTCTTG & TCA-1 (tobacco nuclear protein 1) binding site / T-box-like & TCA-1 & 23 & 16.00 & 2e-005 \\
\hline AGAAAGTG & phyA-induced motifs / Sucrose Responsive Element (SURE)-like & & 23 & 15.00 & $0 e+000$ \\
\hline AАTTATTTA & TATA-box & TBP & 21 & 16.58 & 2e-005 \\
\hline ATTATGAA & JASE1/JASE2-like & AP2/ERF (jasmonate inducible) & 20 & 15.00 & 2e-005 \\
\hline
\end{tabular}

${ }^{1}$ Homology with motifs identified in Arabidopsis and other plant species (TRANSFAC, PLACE and AGRIS databases).

${ }^{2}$ Percent occurrence in critical promoters relative to background sequences.

${ }^{3}$ Total Information Content.

Promoter motifs identified in promoters of RGL2-DOWN, with consensus sequence, name and associated transcription factors. 


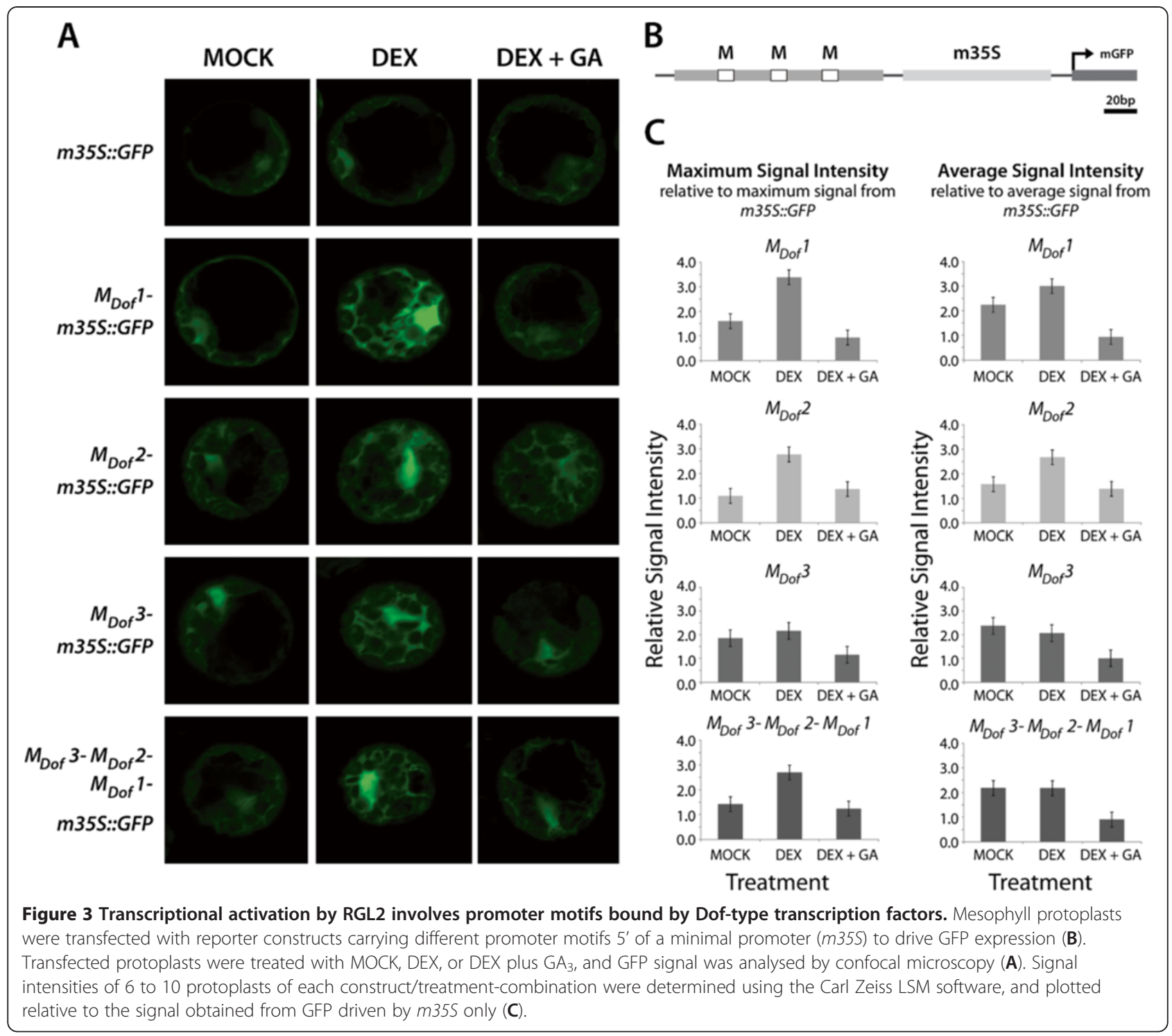

elements are involved in RGL2-mediated transcriptional regulation, we expected to observe changes in GFPsignal intensity; upon DEX-treatment, GFP-signal should increase compared to that observed in MOCK-treated transfected protoplasts, whereas protoplasts treated with DEX plus $\mathrm{GA}_{3}$ should not exhibit different intensities of GFP. Indeed, we were able to observe a significant increase in GFP-intensity in protoplasts containing constructs with two of the three Dof-binding motifs tested (Figure 3A), both with respect to maximum as well as average intensity of fluorescence within a given protoplast (Figure 3C). This increase of GFP-intensity indicates a transcriptional activation by RGL2 (as a complex with other proteins) that is dependent on the presence of these Dof-binding motifs. Interestingly, highest GFPinduction was observed with $\mathrm{M}_{\mathrm{Dof}} 1$, which occurs at the highest frequency (42\%) in our data. Collectively, these results indicate that the predicted motifs in our analysis are indeed cis-regulatory elements involved in RGL2mediated regulation of germination.

RGL2 directly regulates several genes affecting various physiological aspects to inhibit germination

To confirm if a selected few genes from our microarray dataset are directly regulated by RGL2, we performed a Chromatin Immunoprecipitation (ChIP)-quantitative real-time PCR (qRT-PCR) analysis using the 35S::RGL2-GR ga1-3 rga-t2 rgl2-1 transgenic line. Chromatin fragments were isolated from flower buds of 4- to 5-week-old plants $3 \mathrm{~h}$ after treatment with $10 \mu \mathrm{M}$ DEX, using monoclonal anti-GR antibodies. Chromatin isolated in the same manner from Mock-treated flower buds served as control. Since DELLA proteins do not directly bind to DNA, we used two steps of cross-linking during tissue fixation. First, 

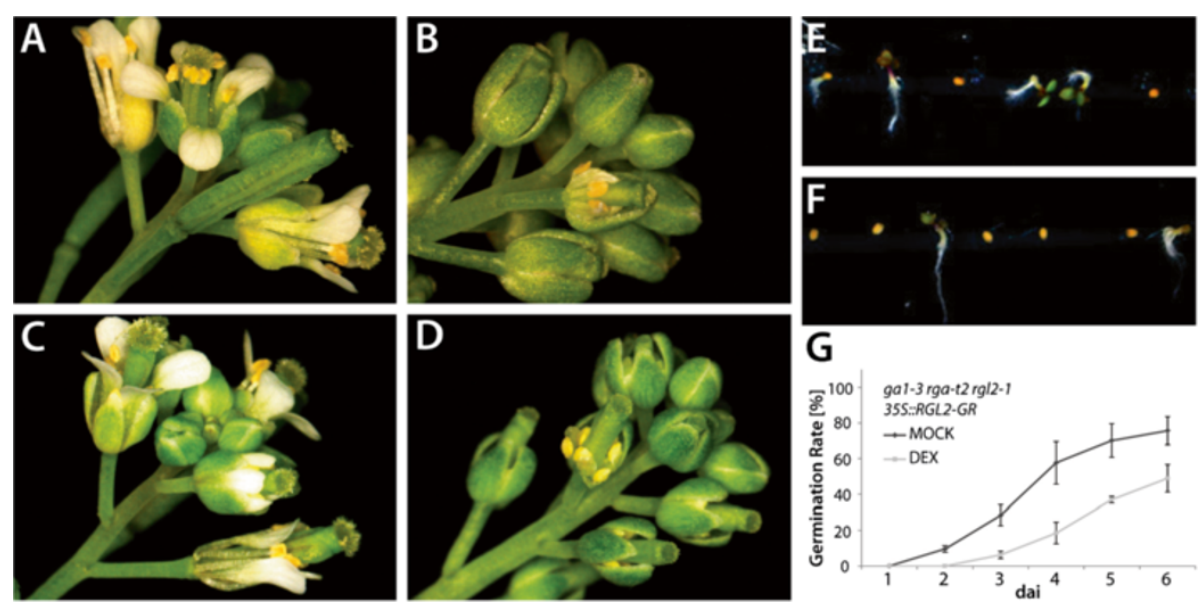

Figure 4 RGL2 tagged with GR is biologically functional. Flower development and seed germination phenotype of ga $1-3 \mathrm{rga}-\mathrm{t} 2 \mathrm{rg} / 2-1$ 35s:: $R G L 2-G R$ without $(\mathbf{A}, \mathbf{E})$ and with $(\mathbf{B}, \mathbf{F})$ DEX-treatment. Without DEX-treatment, RGL2-GR fusion protein is held in the cytoplasm, thus unable to perform its function; the flower phenotype therefore resembles the triple mutant ga -3 rga-t2 $\mathrm{rg} / 2-1$ (C), and seeds readily germinate upon imbibition (E, G). Upon DEX-treatment, RGL2-GR can enter the nucleus and regulate transcription; the flower phenotype is reverted to the double mutant ga 1-3 rga-t2 phenotype with sterile flowers with stunted petals and stamens (D), and germination is significantly delayed and inhibited $(\mathbf{F}, \mathbf{G})$. Two sepals of one flower each have been removed in $(\mathbf{B})$ and $(\mathbf{D})$ to reveal the short petals and stamens. Germination was scored in three biological replicates. Error bars indicate SD. dai - days after imbibition.

we performed a protein-protein cross-linking using disuccinimidyl glutarate (DSG) [54], after which we cross-linked protein with DNA using formaldehyde, followed by chromatin isolation. We tested the efficiency of our protein pull-down by Western Blot, on which we observed a single band for GR-tagged RGL2 (Figure 5A). This not only indicates that we were able to specifically pull down RGL2, but also highlights the efficiency of our additional cross-linking, since no signal from RGL2-GR can be detected post binding. As a further control for our experimental setup, we isolated RNA from flower bud tissue $4 \mathrm{~h}$ after DEX-treatment, and determined expression levels of putative RGL2 target genes identified from our microarray analysis (Figure 5B). Overall, the expression changes of the putative target genes tested overlap with those observed in seeds earlier. However, the changes in expression levels appear to be more moderate compared to those seen in imbibed seeds, with Dof2.1 not showing any change in expression upon DEX-treatment.

For each putative RGL2-target promoter, we designed primers to amplify approximately 90 to $250 \mathrm{bp}$ fragments within the region 1,000 bp upstream of the transcription start site (TSS) (Figure 5C). In isolated chromatin, we determined the enrichment of those promoter fragments over ACTIN2 (ACT2), relative to the control, by qRT-PCR. Overall, we observed a 4- to 6.5-fold enrichment of specific promoter fragments (Figure 5C). From the promoters of downregulated genes tested, both EXPA3 and EXPA8 appear to be bound by the complex involving RGL2; however, the promoter of EXPA8 shows a significantly higher enrichment of all fragments tested, compared to EXPA3. Promoter fragments of the Dof-type transcription factor Dof 2.1 did not show any significant enrichment, which correlates with the lack of transcriptional response in this tissue (Figure 5B). Among promoters of up-regulated genes, the promoter of $A t 2 g 45210$, an as yet uncharacterised gene of the SMALL AUXIN UPREGULATED RNA (SAUR)protein family, shows a strong enrichment.

Selected RGL2-target genes play a role in the regulation of germination in response to gibberellin and abscisic acid

We selected At2g45210, ATHB2 and ATHB5 as representative RGL2 target genes in an attempt to further validate our microarray data. At $2 g 45210$ is a member of the SAUR-gene family of unknown function. It appears as a DELLA-target in both flowers and seeds, and our ChIP data indicate that it is a direct target gene of RGL2. ATHB2 and ATHB5 were chosen as representative homeobox transcription factors that are likely downstream of RGL2 function. $A T H B 2$ appears as up-regulated gene in our microarray only at lower stringency $(\mathrm{p}<0.05)$, but the expression change in seeds of $g a 1-3$ rga- $t 2$ rgl2-1 vs. $g a 1-3$ rga- $t 2$ could be confirmed (Additional file 4). On the other hand, ATHB5 appears to be down-regulated in seeds with stabilised RGL2. Both genes have been characterised previously, with ATHB2 being one of the key transcription factors involved in the regulation of shade avoidance [55], and ATHB5 playing a role in the abscisic acid-mediated repression of germination and seedling root growth [56]. However, no data are available regarding the regulation of 


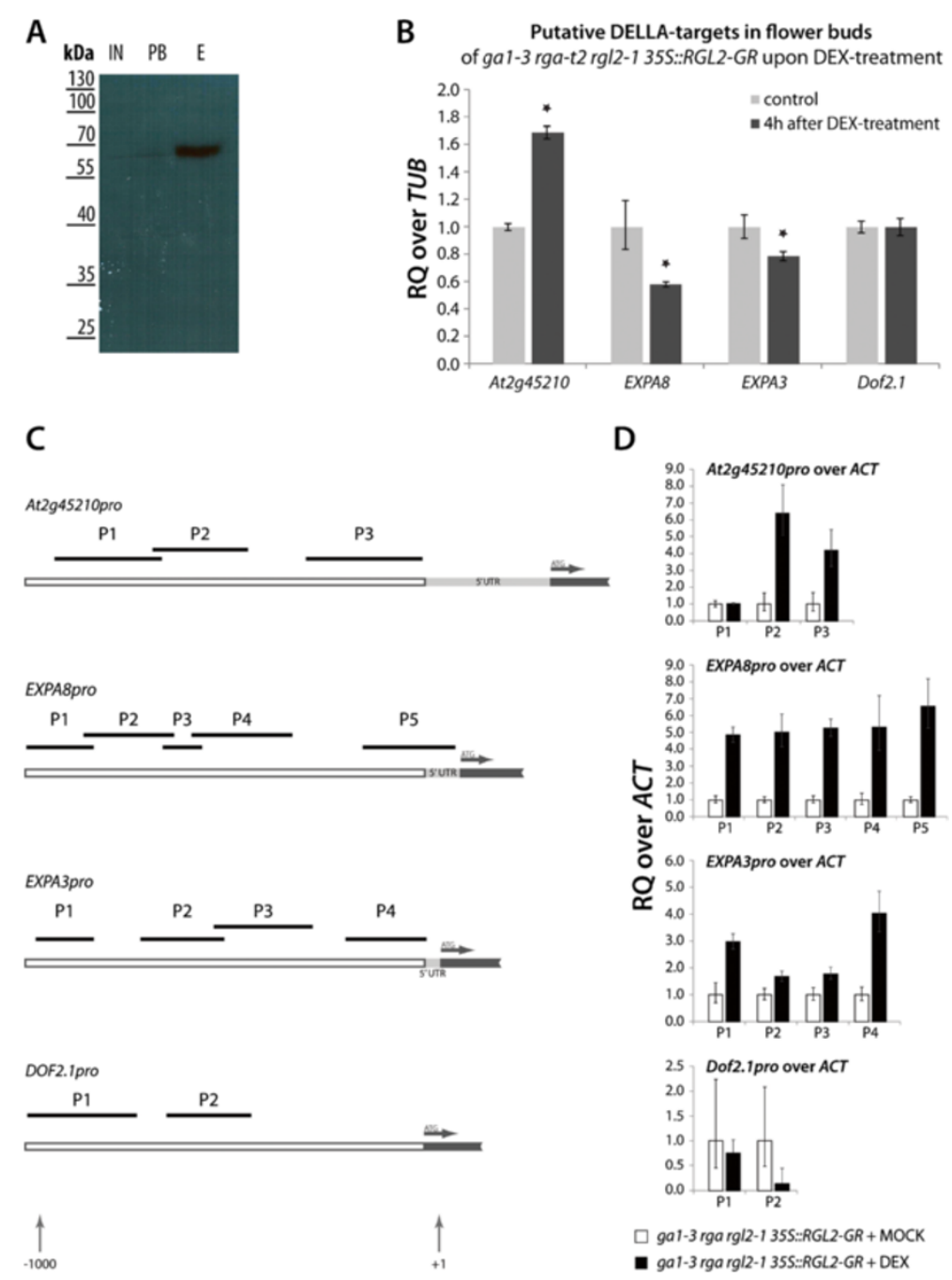

Figure 5 RGL2-GR directly binds to the promoters of various genes. (A) Western Blot using anti-GR antibody on samples at various stages of the protein-chromatin isolation; IN, input, PB, post binding, E, eluted chromatin/protein complex. (B) Relative expression of putative RGL2-/DELLAtarget genes in flower buds of ga1-3 rga-t2 rgl2-1 35S::RGL2-GR 4h after DEX-treatment, relative to Tubulin (TUB). Asterisks indicate a significant difference in expression levels compared to the control $(p<0.05)$. (C) ChIP-qRT-PCR analysis of selected target gene promoters. Promoter regions from $+1,000$ to +1 relative to the transcription start site are depicted, and the position of fragments amplified by qRT-PCR is given as black bars on top. Graphs indicate the relative enrichment of these fragments over ACTIN2 (ACT2) in chromatin isolated from ga 1-3 rga-t2 rgl2-1 35S:::RGL2-GR, 3h after Dex-treatment, compared to chromatin from Mock-treated flower buds. Data were obtained from biological triplicates. $\mathrm{RQ}=$ relative quantity.

germination by $A T H B 2$, or the molecular mechanism by which $A T H B 5$ increases abscisic acid sensitivity in seeds. We were therefore intrigued to explore the possibility of their involvement in gibberellin-mediated germination control.

Significantly higher transcript levels were detected for these three genes in both dry and imbibed seeds, compared to any other tissue of mature plants tested (Figure 6A), suggesting that they could indeed play a role in seed development, maturation and/or germination. Interestingly, expression of At $2 g 45210$ is higher than that of $A T H B 2$ and $A T H B 5$ in all plant tissues tested, and showed a more than 18-fold increase from flower buds (up to developmental stage 13) to open flowers (stage 14).

We obtained T-DNA insertion lines for the three genes, which showed low or no expression of the targeted gene (Figure 6B-D). Germination responses in these mutants were analysed to confirm their involvement in RGL2-, thus gibberellin-mediated germination regulation. Germination rates were examined in response to paclobutrazol (PAC), a gibberellin biosynthesis inhibitor, as well as to abscisic acid, which opposes gibberellin action in germination (Figure 7). As controls, we analysed germination responses of both wild type and the ga1-3 rga-t2 rgl2-1 mutant. The loss of function 


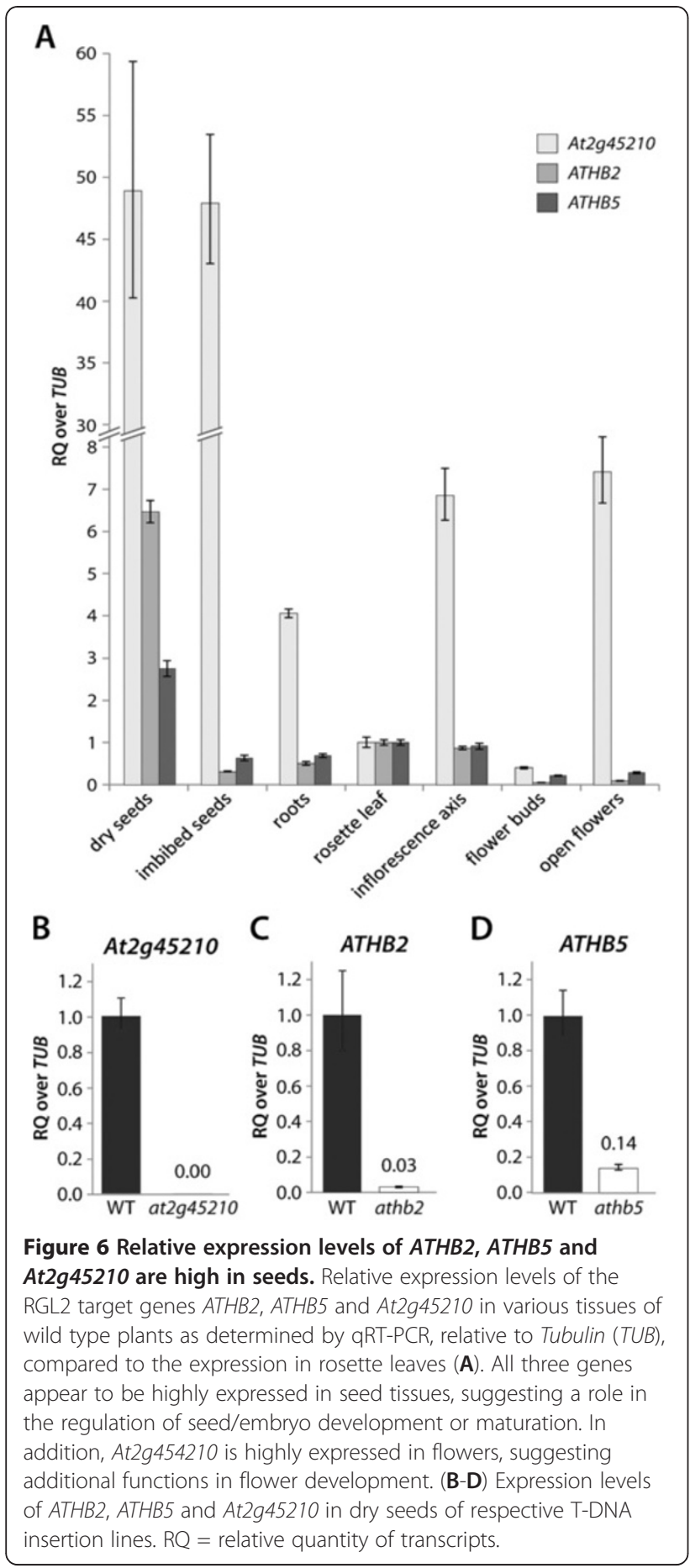

of $R G L 2$ in this triple mutant does not only rescue the non-germinating phenotype of the ga1-3 rga-t2 mutant (Figure 1), but also renders these seeds insensitive to PAC (Figure 7). However, their germination responses to abscisic acid did not differ significantly from those of wild type. Germination of at2g45210 seeds was more severely inhibited than the wild-type seeds by all treatments, indicating that this mutant is hypersensitive to both PAC and abscisic acid with respect to germination. The effect of PAC on seed germination of $a t h b 2$ and athb5 mutants, on the other hand, was not significantly different from that of the wild type. However, athb2 and athb5 mutant seeds differed in their germination response to abscisic acid; at a low concentration $(1 \mu \mathrm{M})$, germination of athb2, but not athb5, is more strongly inhibited than germination of wild-type seeds. However, at higher concentrations of abscisic acid tested, germination of both mutants is inhibited to a greater extent than that of the wild type. The increased sensitivity to abscisic acid of the athb2 and athb5 mutant seeds further prompted us to test whether expression of either gene is responsive to abscisic acid treatment. We therefore determined the relative expression of $A T H B 2$ and $A T H B 5$ in ga1-3 rga-t2 rgl2-1 and ga1-3 rga-t2 seeds that were imbibed in water or abscisic acid (Additional file 4). Interestingly, the transcript levels of the two genes do not differ between the water- and abscisic acid-treatments. In the presence of RGL2, ATHB2 is expressed at a significantly higher level, while $A T H B 5$ shows lower transcript levels.

\section{Discussion}

\section{RGL2-mediated arrest of germination likely induces a} state of secondary dormancy

Due to its immense significance for agriculture germination is one of the most extensively studied fields of plant physiology. Yet, the molecular mechanisms underlying the regulation of germination and dormancy are still largely unknown. In this study, we aimed to elucidate some of the downstream events of the DELLA protein RGL2 in the suppression of germination. Our microarray analysis showed that in non-germinating seeds with stabilised RGL2 protein, genes associated with various stressresponses are enriched among up-regulated genes, along with gibberellin-responsive genes. This is in line with previous transcriptome analyses indicating a high representation of genes related to abscisic acid and stress responses in dormant seeds. On the other hand, as expected, genes associated with embryonic morphogenesis appear to be over-represented in down-regulated genes. More valuable information, however, could be inferred by comparing our gene lists with other available seed- and germination-related microarray data. Overall, the transcriptome of ga1-3 rga-t2 seeds strongly resembles that of dormant seeds, suggesting that seeds with stabilised RGL2 are either unable to leave primary dormancy, or that they are entering a state of secondary dormancy upon imbibition. Thus, RGL2 could be involved in induction or maintenance of seed dormancy. However, we can only speculate if RGL2 maintains primary dormancy or induces secondary dormancy. Nonetheless, it had been determined that gibberellins, thus 


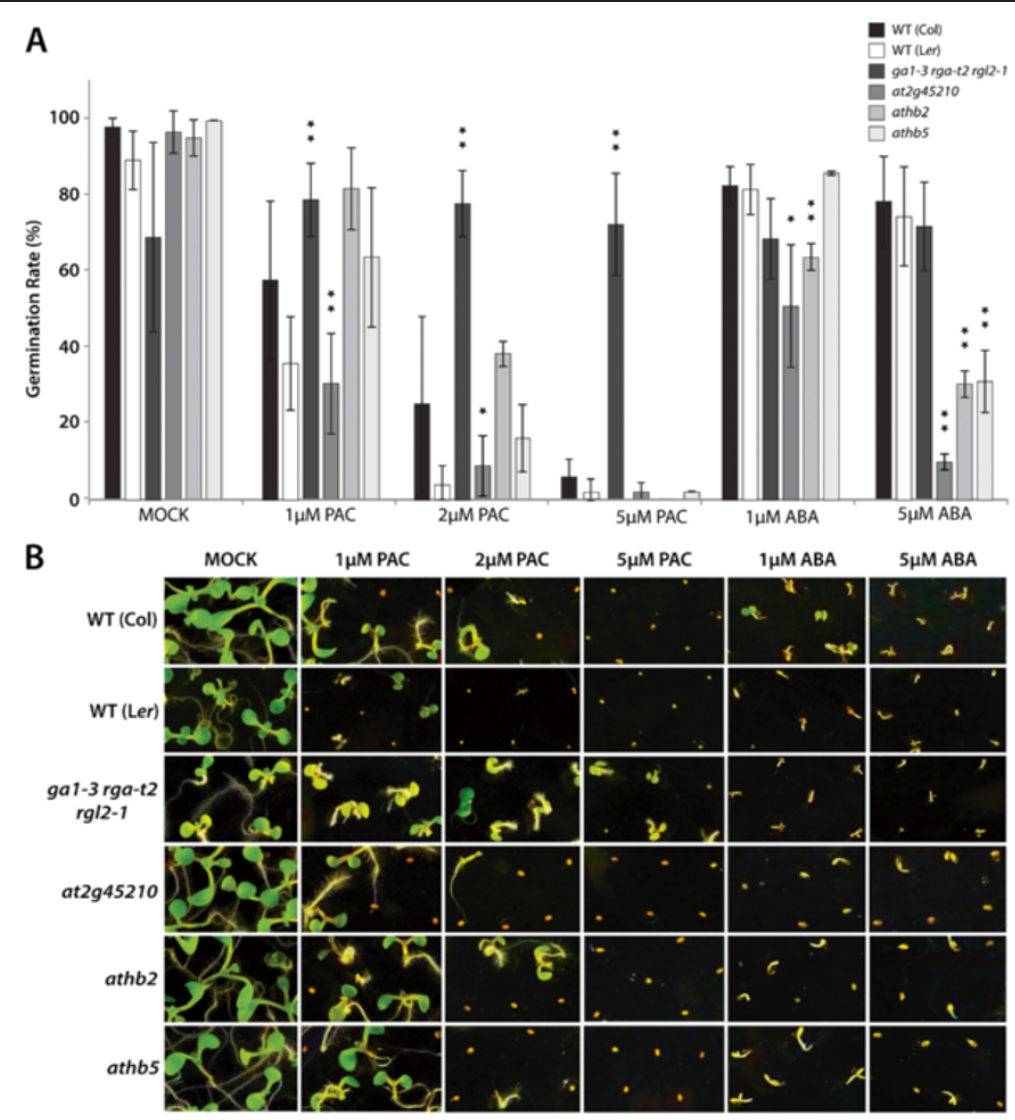

Figure 7 Germination responses are affected in mutants of $A T H B 2, A T H B 5$ and $A t 2 g 45210$. Seeds of wild type and insertion lines were surface sterilised and, without stratification, germinated on 1XMS, supplemented with $0.01 \%$ ethanol (MOCK), 1, 2 or $5 \mu \mathrm{M}$ Paclobutrazol (PAC), 1 or $5 \mu \mathrm{M}$ abscisic acid (ABA). Germination was scored six days after imbibition. Seeds were deemed germinated if the radicle had visibly emerged from the seed coat. Statistical differences were analysed using a two-tailed t-test, and significant differences are indicated by one and two asterisks indicating $p$-values of $p<0.05$ and $p<0.01$, respectively.

RGL2, act late in phase II of the germination process. At this stage seeds have already left primary dormancy, thus have committed to germination. Based on our data, we propose that RGL2 function causes seeds to enter a state of secondary dormancy, in part by directly arresting embryonic growth.

According to our data, RGL2 also seems to positively regulate the expression of a number of transcription factors of MYB-, AP2/ERF-, bHLH- and HD-Zip-types, which are involved in various responses to phytohormones as well as developmental stages. For example, $A T H B 4$ has previously been characterised as a regulator of shade avoidance, being strongly up-regulated by far red light, and modulating the responses to auxins, brassinosteroids and gibberellins in seedlings [57]. MYB111 was shown to be mainly expressed in cotyledons of seedlings, and it plays a role in flavonol accumulation [58]. Two transcription factors have previously been shown to be involved in seed development or germination; WOX2 regulates apical embryo patterning during seed development [59,60], whereas WRKY40, which is strongly up-regulated by abscisic acid, is a transcriptional repressor in abscisic acid and abiotic stress responses [61]. It is tempting to speculate that RGL2 controls far more aspects of seed/embryo development and germination than previously thought, since we observed up-regulation of numerous transcription factors in our microarray analysis. However, the exact transcriptional network governed by RGL2 action, and the relationship between these transcription factors and RGL2 function remains to be further elucidated.

DELLAs have been shown to physically interact with a number of bHLH-type transcription factors, leading to the hypothesis that interaction with bHLH-type of transcription factors is the main underlying molecular mechanism for DELLA function [32-34]. Importantly, it was shown that DELLAs directly interact with PIL5, an inhibitor of seed germination [52]; it is therefore not surprising to find several of its direct target genes in our data. For example, PHYTOCHROME INTERACTING 
FACTOR3-LIKE2 (PIL2) is 3.4-fold up-regulated in our data. It belongs to a light-responsive bHLH transcription factor family, and has been shown to be involved in seed germination responses to red/far red light [62]. Interestingly, PIL2 was also shown to physically interact with RGL2 [34], indicating that RGL2 might be intricately involved in the light-dependent phytochrome-mediated regulation of germination. In RGL2-DOWN, EXPANSIN A8 (EXPA8) and CYSTEINE PROTEINASE1 (CP1), both more than 3.5-fold downregulated, overlap with PIL5 direct targets, amongst others. CP1 belongs to a group of cell wall proteins involved in cell wall modulation and cell growth [63]. Expansins are a group of cell-wall modulating enzymes, many members of which have been shown to be responsive to cold-, abscisic acid- and gibberellin-treatment [reviewed in: 42]. Thus, both genes are involved in weakening cross-links of cell wall components, which allows cell elongation. Therefore, they are considered to be at the "end" of the signalling cascade that leads to cell growth. More importantly, both EXPA8 and CP1 have also been identified as DELLAdependent transcripts [47], further indicating that they could be direct target genes of RGL2.

\section{RGL2-mediated transcriptional regulation should involve the interaction with various classes of transcription factors}

To obtain a better understanding of the regulatory network underlying RGL2 function in the repression of seed germination, we performed an ab initio promoter motif detection in promoters of differentially regulated genes. We identified several motifs that are overrepresented in our data. In promoters of both up- and downregulated genes, we detected a number of GAREs, motifs that are typically bound by transcription factors of the GAMYB-type. GAMYB transcription factors respond to gibberellin signalling, and activate transcription of gibberellin-responsive genes $[64,65]$. This suggests that our microarray data are indeed enriched for genes involved in the gibberellin-mediated regulation of germination, corroborating the quality of our data. It furthermore allows us to hypothesise that RGL2 could interact with GAMYB transcription factors to regulate transcription. Since GARE-like motifs can be found in both RGL2-UP and RGL2-DOWN, this transcriptional regulation is likely mediated by other proteins that form complexes with RGL2. Further investigations into RGL2-interacting proteins are required to clarify this. In addition, motifs associated with the auxin signallingrelated transcription factor ARF1 appear to be enriched in up-regulated genes. This allows us to hypothesise that RGL2-dependent signalling pathway could also interact with auxin signalling in the regulation of embryonic growth.
Furthermore, we observed a strong enrichment of motifs associated with Dof-type transcription factors in the promoters of up-regulated transcripts. Using a protoplast assay, we could confirm that these motifs are indeed involved in the RGL2-mediated transcriptional activation of target genes in planta. It is therefore tempting to speculate that RGL2 might also interact with Dof transcription factors to activate transcription. However, we cannot exclude the possibility that RGL2 binds to and inhibits competitors or inhibitors of Dof proteins, thus allowing Dof proteins to bind to their target promoters and induce transcription, a similar scenario as was reported for DELLA action in the jasmonic acid signalling pathway [36]. Dof proteins are a family of plant-specific transcription factors with some 36 members in Arabidopsis, many of which have been implicated in the regulation of germination. For example, the Dof zinc finger protein Dof Affecting Germination1 (DAG1) and DAG2 have been shown to possess opposing roles in the regulation of germination, with DAG1 inhibiting germination by mediating PIL5 activity as well as directly affecting gibberellin biosynthesis $[66,67]$. Another Dof transcription factor, Dof6, was shown to negatively regulate germination by affecting abscisic acid signalling in seeds [68]. It is therefore likely that Dof proteins play crucial roles in the gibberellin-mediated regulation of germination, and that the enrichment of promoter motifs recognised by Dof transcription factors in our data is of biological significance. It also suggests that either Dof transcription factors themselves or their inhibitors could be binding partners of RGL2. It has been reported that all Dof proteins, likely due to the high similarity of their DNA binding domains, recognise similar target sequences, containing a CTTT consensus core [69]. It was therefore proposed that specificity with regards to spatiotemporal expression and/or interaction with other proteins confers specific functions to each Dof protein [67]. In seeds, it is thus possible that the expression of Dof target genes is specified to phase II of germination through RGL2-action; to shed more light on this complex process of germination, it would be of great interest to identify which specific Dof proteins are involved in the RGL2-mediated repression of germination. Generally, DELLA proteins have been reported to interact with the bHLH class of proteins [32-34] to affect transcription. Our results show that RGL2 likely interacts with various other classes of transcription factors to mediate its response.

RGL2 regulates the expression of various genes to inhibit germination both directly and indirectly

Based on our ChIP-qRT-PCR analyses designed to further understand the molecular events downstream of RGL2, we selected a few genes that were likely direct targets. 
DELLA proteins lack a DNA binding domain, and are thought to regulate transcription through binding to other proteins, most of which are still unknown. We therefore employed a 'double' cross-linking, using DSG and formaldehyde consecutively to capture the whole protein complex bound to chromatin. Previous ChIP assays with DELLA proteins were reported to yield only subtle enrichment (1.3- to 3.5-fold) of target promoter fragments [49]; here, we were able to achieve a significantly higher enrichment (4- to 6.5-fold). This suggests that the application of DSG is a promising method of identifying chromatin regions bound by a protein complex in plants, even if only one of the proteins is known, as has been shown in human cell culture systems [54].

In our data, the RGL2-complex appears to bind to promoters of both EXPA3 and EXPA8, however, promoter fragments of EXPA8 appear more strongly enriched than those of EXPA3. These different levels of enrichment could be due to different binding affinities of the RGL2-complex to these promoters; it could also indicate different binding partners of RGL2 in the complex that binds to DNA. The stronger enrichment of the EXPA8 promoter is in line with our expression analysis showing a more subtle down-regulation of EXPA3 in flower buds of the inducible ga1-3 rga-t2 rgl2-1 35S::RGL2-GR mutant. Previous studies had also shown that EXPA8 expression is more strongly gibberellin-inducible than EXPA3 [42]. Furthermore, EXPA8 appears to be a DELLA-target gene in both flowers and seeds [47]. We therefore propose that the RGL2-complex directly downregulates EXPA8 expression in both flower development and seed germination; thus, RGL2 inhibits both developmental responses by directly constraining cell elongation growth.

Our data further show that the RGL2-complex appears to bind directly to the promoter of a member of the SAUR gene family, At2g45210, whose expression is upregulated in our microarray data. This corroborates further the "updated" gibberellin signalling model [70], which includes not only transcriptional silencing through sequestering of transcription factors by DELLA proteins, but also transcriptional activation by sequestering inhibitors of transcription factors.

Our observation that seed germination in the loss-offunction mutant of At2g45210 exhibits hypersensitivity to abscisic acid and PAC compared to the wild type indicates a possible role for At 2945210 in promoting seed germination. However, its expression is up-regulated by RGL2, an inhibitor of seed germination, and was found to be high in all dormant states of seeds (primary and several secondary dormancy states) analysed by Cadman et al. [71]. This apparent inconsistency of results needs further investigation of this gene's function in seed germination and dormancy. It is possible that it is not directly involved in the inhibition or promotion of seed germination per se. Additionally, we cannot exclude the possibility that its expression is controlled by several other factors not considered here. This is in fact a likely scenario, since its expression pattern only partly overlaps with that of $R G L 2$; it is relatively high in all wild-type tissues tested, whereas RGL2 expression is restricted to imbibed seeds and flower buds [25].

We further investigated the role in the regulation of germination of two homeobox genes, ATHB2 and ATHB5, which were identified in our microarray as up- and downregulated by RGL2, respectively. Both genes have been studied with respect to their roles in other developmental stages; however, no or little data are available to determine their functions in the regulation of seed germination. Interestingly, our seed germination assays appear to reveal different roles for ATHB2 and ATHB5 in germination; while the inhibition of gibberellin biosynthesis with PAC does not result in a germination response different to that of wild type in either mutant, low concentrations of abscisic acid appear to inhibit seed germination more strongly than that of wild type in athb2, but not athb5. However, at high concentrations of abscisic acid, both mutant seeds appear more sensitive to its inhibitory effect. This could indicate that ATHB2 is involved in the abscisic acidmediated regulation of germination, independently of gibberellin. A similar conclusion could be drawn for the role of $A T H B 5$ in the regulation of seed germination; however, the increase in sensitivity of seed germination to abscisic acid is not as high as in athb2. Further germination assays using several intermediate concentrations of abscisic acid would help to determine the range of sensitivity. Interestingly, our data further indicate that expression levels of either ATHB2 or ATHB5 are not affected by abscisic acid treatment. This suggests that the regulation of these two genes is downstream of RGL2, but upstream of the abscisic acid signalling pathway. However, further studies need to be performed, for example, crossing the loss-offunction mutants with various mutants of abscisic acid biosynthesis or signalling, in order to place either gene in the genetic network of gibberellin and abscisic acid signalling. Nevertheless, our results indicate that both these homeobox genes affect seed germination.

\section{Conclusions}

Taken together, our data reveal that the role of RGL2 in the inhibition of germination is complex. We show that RGL2 downregulates the expression of genes encoding cell wall modifying enzymes, viz., $C P 1$ and EXPA8, with at least EXPA8 being directly regulated. Thus, RGL2 directly constrains cell elongation growth to inhibit germination. Our microarray data also indicate that various types of transcription factors are differentially regulated by RGL2, suggesting that RGL2 regulates several aspects 
of seed germination in addition to cell elongation growth, including responses to several phytohormones and light. Our promoter analysis further indicates that RGL2 interacts with various proteins to regulate transcription, including GAMYB, ARF1 and Dof transcription factors. Lastly, we investigated germination responses of three selected target genes to PAC and abscisic acid. We show that sensitivity of germination to at least one of these treatments is increased in these mutants, which not only indicates that all of these genes are in some way involved in the regulation of germination, but also validates the quality and reliability of our data. However, more work needs to be done to understand the mechanism of regulation of germination, and to shed more light on the genetic network underlying the complex process of seed germination in which RGL2 is but one player.

\section{Methods}

\section{Plant materials, plant transformation and growth} conditions

Arabidopsis thaliana accessions used in this study were either Columbia (Col-0) or Landsberg erecta (Ler). Plants were grown in a growth chamber with a 16-hlight/8-h-dark cycle, at $23^{\circ} \mathrm{C}$ and $75 \% \mathrm{RH}$ for generation of transgenic plants and seed collection. Plant transformation was performed as described by Clough and Bent [72] using Agrobacterium tumefaciens strain GV3101: pMP90.

All gibberellin-related mutants described here are in Ler background; ga1-3 rga-t2 and ga1-3 rga-t2 rgl2-1 were described earlier [30]. The inducible transgenic plants ga1-3 rga-t2 $\mathrm{rgl2}-1$ 35S::RGL2-GR were generated by transforming ga1-3 rga-t2 rgl2-1 plants with the binary vector harbouring the $35 S:: R G L 2-G R$ cassette. Insertion lines for At2g45210 (SALK_142329), ATHB2 (SALK_106790C) and ATHB5 (SALK_122765) were obtained from the ABRC seed stock, and are in Columbia (Col-0) background. Homozygous plants were identified by genotyping with primers designed using the $\mathrm{T}$ DNA primer design tool (http://signal.salk.edu/tdnaprimers.2.html; Additional file 5).

For germination assays, seeds were surface sterilised in $75 \%$ ethanol and $15 \%$ commercial bleach, followed by at least five rinses with sterile water. Aseptic seeds were placed on $1 \mathrm{xMS}$ medium, pH 5.7 with $0.5 \%$ Gelrite, supplemented with either of the following: $0.01 \%$ ethanol (MOCK), $10 \mu \mathrm{M}$ gibberellic acid $3\left(\mathrm{GA}_{3}\right), 1 \mu \mathrm{M}, 2 \mu \mathrm{M}$ or $5 \mu \mathrm{M}$ paclobutrazol (PAC), $1 \mu \mathrm{M}$ or $5 \mu \mathrm{M}$ abscisic acid, $10 \mu \mathrm{M}$ dexamethasone (DEX). To analyse germination inhibition by DEX of the inducible gal-3 rga-t2 rgl2-1 35S::RGL2-GR mutant, aseptic seeds were vacuuminfiltrated with $30 \mu \mathrm{M}$ DEX prior to being placed on MS plates. All plates were incubated at $22^{\circ} \mathrm{C}$ with a 16 -h- light/8-h-dark cycle. Germination was scored up to six days after imbibition. Seeds were deemed germinated if the radicle had visibly protruded through the seed coat.

\section{Microarray experiments and data analysis}

Total RNA was isolated from seeds imbibed in water at $4^{\circ} \mathrm{C}$ in the dark for five days, according to Vicient and Delseny [73], with slight modifications. For each isolation 50mg (dry weight) seeds were used. Crude RNA was purified by extractions with chloroform, phenol, phenol:chloroform:isoamyl alcohol (25:24:1) and chloroform:isoamyl alcohol (24:1), once each. After precipitation, RNA was dissolved in 30 to $50 \mu \mathrm{l}$ water.

Total RNA was sent to Genotypic Technology [P] Ltd., Bangalore, India for microarray analysis (Project No. GT-537_E), including RNA quality control using Bioanalyser, reverse transcription and labelling, single colour hybridisation onto Agilent Arabidopsis 4×44k Array, preliminary data analysis with GeneSpring GX version 10.0 and Excel, data normalisation using Percentile Shift Normalisation and Normalisation to Specific Samples. Microarray data were deposited in the Gene Expression Omnibus (GEO; http://www.ncbi.nlm.nih.gov/projects/ geo), accession number GSE40485.

\section{Chromatin immunoprecipitation}

Chromatin Immunoprecipitation (ChIP) was performed using flower buds of 3-4-week-old ga1-3 rga-t2 rgl2-1 35S::RGL2-GR plants. Plants were sprayed with either of the following: $0.01 \%$ ethanol (MOCK), or $10 \mu \mathrm{M} \mathrm{DEX,}$ and flower buds were harvested $3 \mathrm{~h}$ after treatment. ChIP was performed according to Kaufmann et al. [74], with minor changes. We included an additional step of protein-protein cross-linking using $10 \mathrm{mM}$ disuccinimidyl glutarate (DSG) [54], prior to cross-linking of DNAprotein complexes by formaldehyde. All centrifugation steps were performed at maximum speed $(\sim 17,000 \mathrm{xg})$. For the detection of RGL2-GR, total extracts, flow through and eluates prior to proteinase $\mathrm{K}$ treatment were resolved under reducing conditions on 12\% SDS/ polyacrylamide gel, and proteins were transferred onto polyvinylidene difluoride (PVDF) membranes (Bio-Rad Laboratories). PVDF membranes were blocked by incubation with $5 \%$ milk powder in Phosphate-buffered saline $+0.05 \%$ Tween 20 (PBS-T) over night, followed by incubation with monoclonal mouse anti-GR antibody $(1: 1,000)$ at room temperature for $2 \mathrm{~h}$. After washing with PBS-T, membranes were incubated with secondary antibody, horseradish peroxidase- (HRP-) conjugated rabbit anti-mouse antibody $(1: 10,000)$ for $2 \mathrm{~h}$ at room temperature. Membranes were then washed four times with PBS-T, and immune complexes were detected on x-ray film (Fuji medical x-ray film) using the Enhanced 
Chemiluminescence (ECL) Detection kit according to the manufacturer's instructions (Amersham Pharmacia).

\section{Quantitative real-time PCR (qRT-PCR)}

Comparative analysis of selected genes was performed by qRT-PCR. Reactions were performed on cDNA, prepared from RNA of various Arabidopsis tissues using 'MAXIMA ${ }^{\circledR}$ First Strand cDNA Synthesis Kit' (Fermentas), with 'KAPA SYBR ${ }^{\circledR}$ FAST qPCR Kit' (KAPA Biosystems) using the 'StepOne ${ }^{\mathrm{TM}}$ Real-Time PCR Systems' (Applied Biosystems). All qRT-PCR data were generated from biological duplicates. Relative quantification of expression was determined using 'StepOne Software' (v2.1). For a list of primers, see Additional file 5.

\section{$A b$ initio promoter analysis}

The entire sets of up-regulated (253) and downregulated (354) genes, respectively, were analysed for enrichment of promoter motifs. Sequences of promoter regions $(-1,000$ to $+200 \mathrm{nt}$ relative to TSS) were extracted from our in-house promoter sequence database. Over-represented motifs were detected using the Dragon Motif Builder algorithm with EM2 option [75], and thirty motifs of 8 to 10 nucleotides were detected each with a threshold value of 0.95 . Random promoter sequences were used for background subtraction of random motif occurrence. Significant motifs were selected based on a threshold occurrence of more than $20 \%$, and motif classes were identified by significant matches with TRANSFAC [76], PLACE [77] and AGRIS [78,79] databases.

\section{Plasmid construction, protoplast isolation and transfection}

For promoter motif analysis, a minimal promoter (90bp of CaMV 35S promoter) was amplified with SmaI and BamHI restriction sites, and cloned into pGreen (HY105 backbone) containing mGFP (cloned with SpeI and $\mathrm{XbaI})$, to generate pGreen-m35S::GFP. Synthetic promoter constructs containing three tandem copies of selected promoter motifs were generated by overlapping PCR using synthesised oligonucleotides (see Additional file 5). The generated 126bp-fragments were cloned into the binary vector using HindIII and PstI restriction sites, 5 ' of the minimal promoter in pGreen-m35S::GFP.

Leaf mesophyll protoplasts were isolated from 3- to 4week-old wild-type Arabidopsis (Col-0) plants following the protocol described in Yoo et al. [80]. For each transfection, 10 to $15 \mu \mathrm{g}$ of plasmid DNA was used, and treatment occurred $30 \mathrm{~min}$ after transfection with either of the following: $0.01 \%$ ethanol (MOCK), 10 $\mu \mathrm{M}$ DEX, $10 \mu \mathrm{M}$ DEX plus $10 \mu \mathrm{M} \mathrm{GA}_{3}$. Images were acquired four to six hours after transfection using a Carl Zeiss Axiovert 200M confocal laser microscope (http://www.zeiss. de/axiovert200) with excitation at $488 \mathrm{~nm}$. For GFP detection, one channel was configured between 505 and $530 \mathrm{~nm}$. All images were recorded with the same detection settings. Average and maximum signal intensity of 6 to 10 selected protoplasts was determined using the Carl Zeiss LSM software (ver. 4.0).

\section{Additional files}

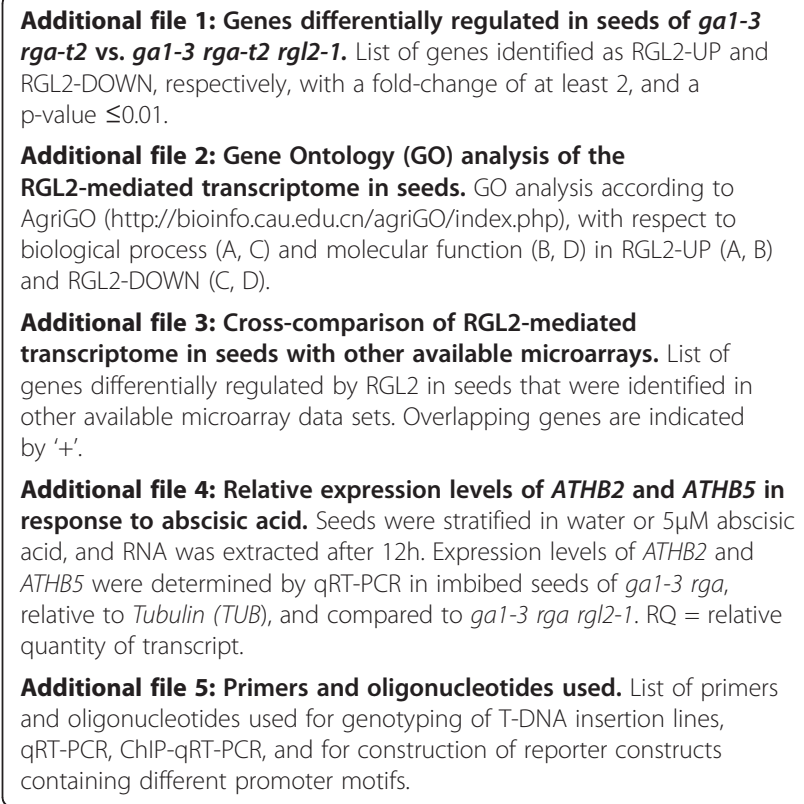

Additional file 3: Cross-comparison of RGL2-mediated transcriptome in seeds with other available microarrays. List of genes differentially regulated by RGL2 in seeds that were identified in other available microarray data sets. Overlapping genes are indicated by ' + '.

Additional file 4: Relative expression levels of ATHB2 and ATHB5 in response to abscisic acid. Seeds were stratified in water or $5 \mu \mathrm{M}$ abscisic acid, and RNA was extracted after 12h. Expression levels of ATHB2 and ATHB5 were determined by qRT-PCR in imbibed seeds of gal-3 rga, relative to Tubulin (TUB), and compared to ga1-3 rga rg/2-1. RQ = relative quantity of transcript.

Additional file 5: Primers and oligonucleotides used. List of primers and oligonucleotides used for genotyping of T-DNA insertion lines, qRT-PCR, ChIP-qRT-PCR, and for construction of reporter constructs containing different promoter motifs.

\section{Abbreviations}

ChIP: chromatin immunoprecipitation; DEX: dexamethasone; Dof: DNA binding with one finger; EXPA8: Expansin A 8; GA: gibberellic acid; GFP: green fluorescence protein; RGL2: RGA-like 2; qRT-PCR: quantitative real-time PCR.

\section{Competing interests}

There are no competing interests.

\section{Authors' contributions}

PS designed and performed all experiments, including all statistical and bioinformatics analyses, and wrote the manuscript. PR optimised and performed RNA extractions, ChIP and qRT-PCR experiments. BM performed the $a b$ initio promoter analysis. ELT generated the inducible line. HY helped in designing the project and writing the manuscript. PPK was responsible for overall supervision of experimental design, data analysis, and writing the manuscript. All authors approved the final manuscript.

\section{Acknowledgements}

This project was partially supported by grant number R-154-000-154-720 from the National University of Singapore (NUS). PS and PR were recipients of research scholarship from NUS.

\section{Author details}

${ }^{1}$ Department of Biological Sciences, Faculty of Science, National University of Singapore, Singapore 117543, Singapore. ${ }^{2}$ Department of Chemical and Biomolecular Engineering, National University of Singapore, Singapore 117576, Singapore. ${ }^{3}$ Temasek Life Sciences Laboratory, National University of Singapore, 1 Research Link, Singapore 117604, Singapore.

Received: 22 May 2012 Accepted: 1 October 2012

Published: 4 October 2012 


\section{References}

1. Richards DE, King KE, Ait-Ali T, Harberd NP: How gibberellin regulates plant growth and development: A molecular genetic analysis of gibberellin signaling. Annual Review of Plant Physiology and Plant Molecular Biology 2001, 52:67-88.

2. Olszewski N, Sun TP, Gubler F: Gibberellin signaling: biosynthesis, catabolism, and response pathways. Plant Cell 2002, 14(Suppl):S61-80.

3. Peng J, Harberd NP: The role of GA-mediated signalling in the control of seed germination. Curr Opin Plant Biol 2002, 5(5):376-381.

4. Sun TP, Gubler F: Molecular mechanism of gibberellin signaling in plants. Annu Rev Plant Biol 2004, 55:197-223.

5. Koornneef $\mathrm{M}$, Veen JH: Induction and analysis of gibberellin sensitive mutants in Arabidopsis thaliana (L.) heynh. Theor Appl Genet 1980, 58(6):257-263.

6. Sun TP, Kamiya Y: The Arabidopsis GA1 locus encodes the cyclase entkaurene synthetase A of gibberellin biosynthesis. Plant Cell 1994, 6(10):1509-1518.

7. Hartweck LM: Gibberellin signaling. Planta 2008, 229(1):1-13.

8. Schwechheimer C: Understanding gibberellic acid signaling-are we there yet? Curr Opin Plant Biol 2008, 11(1):9-15.

9. Hartweck LM, Olszewski NE: Rice GIBBERELLIN INSENSITIVE DWARF1 is a gibberellin receptor that illuminates and raises questions about GA signaling. Plant Cell 2006, 18(2):278-282.

10. Ueguchi-Tanaka M, Ashikari M, Nakajima M, Itoh H, Katoh E, Kobayashi M, Chow TY, Hsing YI, Kitano H, Yamaguchi I, et al: GIBBERELLIN INSENSITIVE DWARF1 encodes a soluble receptor for gibberellin. Nature 2005, 437(7059):693-698.

11. Griffiths J, Murase K, Rieu I, Zentella R, Zhang Z-L, Powers SJ, Gong F, Phillips AL, Hedden P, Sun T-p, et al: Genetic characterization and functional analysis of the GID1 gibberellin receptors in Arabidopsis. Plant Cell 2006, 18(12):3399-3414.

12. Fu X, Richards DE, Ait-ali T, Hynes LW, Ougham H, Peng J, Harberd NP. Gibberellin-mediated proteasome-dependent degradation of the barley DELLA protein SLN1 repressor. Plant Cell 2002, 14(12):3191-3200.

13. Hussain A, Cao D, Cheng H, Wen Z, Peng J: Identification of the conserved serine/threonine residues important for gibberellin-sensitivity of Arabidopsis RGL2 protein. Plant J 2005, 44(1):88-99.

14. Itoh $\mathrm{H}$ : The gibberellin signaling pathway is regulated by the appearance and disappearance of SLENDER RICE1 in nuclei. Plant Cell 2002, 14(1):57-70.

15. Silverstone AL, Jung H-S, Dill A, Kawaide H, Kamiya Y, Sun T-p: Repressing a repressor: gibberellin-induced rapid reduction of the RGA protein in Arabidopsis. Plant Cell 2001, 13(7):1555-1566.

16. Dill A, Thomas SG, Hu J, Steber CM, Sun TP: The Arabidopsis F-box protein SLEEPY1 targets gibberellin signaling repressors for gibberellin-induced degradation. Plant Cell 2004, 16(6):1392-1405.

17. MCGinnis KM, Thomas SG, Soule JD, Strader LC, Zale JM, Sun TP, Steber CM: The Arabidopsis SLEEPY1 gene encodes a putative F-box subunit of an SCF E3 ubiquitin ligase. Plant Cell 2003, 15(5):1120-1130.

18. Sasaki A, Itoh H, Gomi K, Ueguchi-Tanaka M, Ishiyama K, Kobayashi M, Jeong $\mathrm{DH}, \mathrm{An} \mathrm{G}$, Kitano H, Ashikari M, et al: Accumulation of phosphorylated repressor for gibberellin signaling in an F-box mutant. Science 2003, 299(5614):1896-1898.

19. Fu X, Richards DE, Fleck B, Xie D, Burton N, Harberd NP: The Arabidopsis mutant sleepy $1^{\text {gar2-1 }}$ protein promotes plant growth by increasing the affinity of the $\mathrm{SCF}^{\mathrm{SLY} Y}$ E3 ubiquitin ligase for DELLA protein substrates. Plant Cell 2004, 16(6):1406-1418.

20. Pysh LD, Wysocka-Diller JW, Camilleri C, Bouchez D, Benfey PN: The GRAS gene family in Arabidopsis: sequence characterization and basic expression analysis of the SCARECROW-like genes. Plant J 1999, 18(1):111-119.

21. Boss PK, Thomas MR: Association of dwarfism and floral induction with a grape 'green revolution' mutation. Nature 2002, 416(6883):847-850.

22. Chandler PM, Marion-Poll A, Ellis M, Gubler F: Mutants at the Slender1 locus of barley cv Himalaya. Molecular and physiological characterization. Plant Physiol 2002, 129(1):181-190.

23. Peng J, Richards DE, Hartley NM, Murphy GP, Devos KM, Flintham JE, Beales J, Fish LJ, Worland AJ, Pelica F, et al: 'Green revolution' genes encode mutant gibberellin response modulators. Nature 1999, 400(6741):256-261

24. Dill A, Sun T: Synergistic derepression of gibberellin signaling by removing RGA and GAl function in Arabidopsis thaliana. Genetics 2001, 159(2):777-785.
25. Lee S, Cheng H, King KE, Wang W, He Y, Hussain A, Lo J, Harberd NP, Peng J: Gibberellin regulates Arabidopsis seed germination via RGL2, a GAI/RGAlike gene whose expression is up-regulated following imbibition. Genes Dev 2002, 16(5):646-658.

26. Wen CK, Chang C: Arabidopsis RGL1 encodes a negative regulator of gibberellin responses. Plant Cell 2002, 14(1):87-100.

27. Cheng H, Qin L, Lee S, Fu X, Richards DE, Cao D, Luo D, Harberd NP, Peng J: Gibberellin regulates Arabidopsis floral development via suppression of DELLA protein function. Development 2004, 131(5):1055-1064.

28. King KE, Moritz T, Harberd NP: Gibberellins are not required for normal stem growth in Arabidopsis thaliana in the absence of GAI and RGA. Genetics 2001, 159(2):767-776.

29. Tyler L, Thomas SG, Hu J, Dill A, Alonso JM, Ecker JR, Sun TP: DELLA proteins and gibberellin-regulated seed germination and floral development in Arabidopsis. Plant Physiol 2004, 135(2):1008-1019.

30. Yu H, Ito T, Zhao Y, Peng J, Kumar P, Meyerowitz EM: Floral homeotic genes are targets of gibberellin signaling in flower development. Proc Natl Acad Sci 2004, 101(20):7827-7832

31. Cao D, Hussain A, Cheng H, Peng J: Loss of function of four DELLA genes leads to light- and gibberellin-independent seed germination in Arabidopsis. Planta 2005, 223(1):105-113.

32. de Lucas M, Daviere J-M, Rodriguez-Falcon M, Pontin M, Iglesias-Pedraz JM, Lorrain S, Fankhauser C, Blazquez MA, Titarenko E, Prat S: A molecular framework for light and gibberellin control of cell elongation. Nature 2008, 451(7177):480-484

33. Feng S, Martinez C, Gusmaroli G, Wang Y, Zhou J, Wang F, Chen L, Yu L, Iglesias-Pedraz JM, Kircher S, et al: Coordinated regulation of Arabidopsis thaliana development by light and gibberellins. Nature 2008, 451(7177):475-479.

34. Gallego-Bartolomé J, Minquet EG, Marín JA, Prat S, Blázquez MA, Alabadí D: Transcriptional diversification and functional conservation between DELLA proteins in Arabidopsis. Mol Biol Evol 2010, 27(6):1247-1256.

35. Davière $J M$, de Lucas $M$, Prat $\mathrm{S}$ : Transcriptional factor interaction: a central step in DELLA function. Curr Opin Genet Dev 2008, 18(4):295-303.

36. Hou X, Lee LY, Xia K, Yan Y, Yu H: DELLAs modulate jasmonate signaling via competitive binding to JAZs. Dev Cell 2010, 19(6):884-894.

37. Richter R, Behringer C, Muller IK, Schwechheimer C: The GATA-type transcription factors GNC and GNL/CGA1 repress gibberellin signaling downstream from DELLA proteins and PHYTOCHROME-INTERACTING FACTORS. Genes Dev 2010, 24(18):2093-2104.

38. Toledo-Ortiz G, Huq E, Rodriguez-Concepcion M: Direct regulation of phytoene synthase gene expression and carotenoid biosynthesis by phytochromeinteracting factors. Proc Natl Acad Sci 2010, 107(25):11626-11631.

39. Bewley JD: Seed germination and dormancy. Plant Cell 1997, 9(7):1055-1066.

40. Bewley JD, Black M: Seeds: Physiology of Development and Germination. 2nd edition. New York: Springer; 1994

41. Ogawa M, Hanada A, Yamauchi Y, Kuwahara A, Kamiya Y, Yamaguchi S: Gibberellin biosynthesis and response during Arabidopsis seed germination. Plant Cell 2003, 15(7):1591-1604.

42. Weitbrecht $K$, Muller $K$, Leubner-Metzger G: First off the mark: early seed germination. J Exp Bot 2011, 62(10):3289-3309.

43. Piskurewicz U, Jikumaru Y, Kinoshita N, Nambara E, Kamiya Y, Lopez-Molina L: The gibberellic acid signaling repressor RGL2 inhibits Arabidopsis seed germination by stimulating abscisic acid synthesis and ABI5 activity. Plant Cell 2008, 20(10):2729-2745.

44. Piskurewicz U, Tureckova V, Lacombe E, Lopez-Molina L: Far-red light inhibits germination through DELLA-dependent stimulation of ABA synthesis and ABI3 activity. EMBO J 2009, 28(15):2259-2271.

45. Lee KP, Piskurewicz U, Tureckova V, Strnad M, Lopez-Molina L: A seed coat bedding assay shows that RGL2-dependent release of abscisic acid by the endosperm controls embryo growth in Arabidopsis dormant seeds. Proc Natl Acad Sci 2010, 107(44):19108-19113.

46. Holdsworth MJ, Bentsink L, Soppe WJJ: Molecular networks regulating Arabidopsis seed maturation, after-ripening, dormancy and germination. New Phytol 2008, 179(1):33-54.

47. Cao D, Cheng $H$, Wu W, Soo HM, Peng J: Gibberellin mobilizes distinct DELLA-dependent transcriptomes to regulate seed germination and floral development in Arabidopsis. Plant Physiol 2006, 142(2):509-525.

48. Yamauchi Y, Ogawa M, Kuwahara A, Hanada A, Kamiya Y, Yamaguchi S: Activation of gibberellin biosynthesis and response pathways by low 
temperature during imbibition of Arabidopsis thaliana seeds. Plant Cell 2004, 16(2):367-378.

49. Zentella R, Zhang Z-L, Park M, Thomas SG, Endo A, Murase K, Fleet CM, Jikumaru Y, Nambara E, Kamiya Y, et al: Global analysis of DELLA direct targets in early gibberellin signaling in Arabidopsis. Plant Cell 2007, 19(10):3037-3057.

50. Carrera E, Holman T, Medhurst A, Peer W, Schmuths H, Footitt S, Theodoulou FL, Holdsworth MJ: Gene expression profiling reveals defined functions of the ATP-binding cassette transporter COMATOSE late in phase II of germination. Plant Physio/ 2007, 143(4):1669-1679.

51. Oh E, Kim J, Park E, Kim JI, Kang C, Choi G: PIL5, a phytochrome-interacting basic helix-loop-helix protein, is a key negative regulator of seed germination in Arabidopsis thaliana. Plant Cell 2004, 16(11):3045-3058.

52. Oh E, Kang H, Yamaguchi S, Park J, Lee D, Kamiya Y, Choi G: Genome-wide analysis of genes targeted by PHYTOCHROME INTERACTING FACTOR 3LIKE5 during seed germination in Arabidopsis. Plant Cell 2009, 21(2):403-419.

53. Wagner D, Sablowski RW, Meyerowitz EM: Transcriptional activation of APETALA1 by LEAFY. Science 1999, 285(5427):582-584.

54. Nowak D, Tian B, Brasier A: Two-step cross-linking method for identification of NF-KB gene network by chromatin immunoprecipitation. Biotechniques 2005, 39(5):715-725.

55. Lorrain S, Allen T, Duek PD, Whitelam GC, Fankhauser C: Phytochromemediated inhibition of shade avoidance involves degradation of growthpromoting bHLH transcription factors. Plant J 2008, 53(2):312-323.

56. Johannesson H, Wang Y, Hanson J, Engstrom P: The Arabidopsis thaliana homeobox gene $A T H B 5$ is a potential regulator of abscisic acid responsiveness in developing seedlings. Plant Mol Biol 2003, 51(5):719-729.

57. Sorin C, Salla-Martret M, Bou-Torrent J, Roig-Villanova I, Martinez-Garcia JF: ATHB4, a regulator of shade avoidance, modulates hormone response in Arabidopsis seedlings. Plant J 2009, 59(2):266-277.

58. Stracke R, Ishihara H, Huep G, Barsch A, Mehrtens F, Niehaus K, Weisshaar B: Differential regulation of closely related R2R3-MYB transcription factors controls flavonol accumulation in different parts of the Arabidopsis thaliana seedling. Plant J 2007, 50(4):660-677.

59. Breuninger H, Rikirsch E, Hermann M, Ueda M, Laux T: Differential expression of WOX genes mediates apical-basal axis formation in the Arabidopsis embryo. Dev Cell 2008, 14(6):867-876.

60. Wu X, Chory J, Weigel D: Combinations of WOX activities regulate tissue proliferation during Arabidopsis embryonic development. Dev Biol 2007, 309(2):306-316.

61. Chen H, Lai Z, Shi J, Xiao Y, Chen Z, Xu X: Roles of Arabidopsis WRKY18, WRKY40 and WRKY60 transcription factors in plant responses to abscisic acid and abiotic stress. BMC Plant Biol 2010, 10:281.

62. Penfield S, Josse E-M, Halliday K: A role for an alternative splice variant of PIF6 in the control of Arabidopsis primary seed dormancy. Plant Mol Biol 2010, 73(1):89-95.

63. Irshad M, Canut $\mathrm{H}$, Borderies $\mathrm{G}$, Pont-Lezica $\mathrm{R}$, Jamet E: A new picture of cell wall protein dynamics in elongating cells of Arabidopsis thaliana: confirmed actors and newcomers. BMC Plant Biol 2008, 8:94.

64. Gubler F, Kalla R, Roberts JK, Jacobsen JV: Gibberellin-regulated expression of a Myb gene in barley aleurone cells: evidence for Myb transactivation of a high-pl alpha-amylase gene promoter. Plant Cell 1995, 7(11):1879-1891.

65. Gubler F, Raventos D, Keys M, Watts R, Mundy J, Jacobsen JV: Target genes and regulatory domains of the GAMYB transcriptional activator in cereal aleurone. Plant J 1999, 17(1):1-9.

66. Gabriele S, Rizza A, Martone J, Circelli P, Costantino P, Vittorioso P: The Dof protein DAG1 mediates PIL5 activity on seed germination by negatively regulating GA biosynthetic gene AtGA3ox1. Plant J 2010, 61(2):312-323.

67. Gualberti G, Papi M, Bellucci L, Ricci I, Bouchez D, Camilleri C, Costantino P, Vittorioso P: Mutations in the Dof zinc finger genes DAG2 and DAG1 influence with opposite effects the germination of Arabidopsis seeds. Plant Cell 2002, 14(6):1253-1263.

68. Rueda-Romero P, Barrero-Sicilia C, Gomez-Cadenas A, Carbonero P, OnateSanchez L: Arabidopsis thaliana DOF6 negatively affects germination in non-after-ripened seeds and interacts with TCP14. J Exp Bot 2011, 63(5):1937-1949.

69. Yanagisawa S, Schmidt RJ: Diversity and similarity among recognition sequences of Dof transcription factors. Plant J 1999, 17(2):209-214

70. Gao XH, Xiao SL, Yao QF, Wang YJ, Fu XD: An updated GA signaling 'relief of repression' regulatory model. Mol Plant 2011, 4(4):601-606.
71. Cadman CSC, Toorop PE, Hilhorst HWM, Finch-Savage WE: Gene expression profiles of Arabidopsis Cvi seeds during dormancy cycling indicate a common underlying dormancy control mechanism. Plant J 2006, 46(5):805-822.

72. Clough SJ, Bent AF: Floral dip: a simplified method for Agrobacteriummediated transformation of Arabidopsis thaliana. Plant J 1998, 16(6):735-743.

73. Vicient CM, Delseny M: Isolation of total RNA from Arabidopsis thaliana seeds. Anal Biochem 1999, 268(2):412-413.

74. Kaufmann K, Muiño JM, Østerås M, Farinelli L, Krajewski P, Angenent GC: Chromatin immunoprecipitation (ChIP) of plant transcription factors followed by sequencing (ChIP-SEQ) or hybridization to whole genome arrays (ChIP-CHIP). Nat Protoc 2010, 5(3):457-472.

75. Huang E, Yang L, Chowdhary R, Kassim A, Bajic VB (Eds): An algorithm for ab initio DNA motif detection. World Scientific; 2005.

76. Matys V, Fricke E, Geffers R, Gossling E, Haubrock M, Hehl R, Hornischer K, Karas D, Kel AE, Kel-Margoulis OV, et al: TRANSFAC: transcriptional regulation, from patterns to profiles. Nucleic Acids Res 2003, 31(1):374-378.

77. Higo K, Ugawa Y, Iwamoto M, Korenaga T: Plant cis-acting regulatory DNA elements (PLACE) database: 1999. Nucleic Acids Res 1999, 27(1):297-300.

78. Davuluri RV, Sun H, Palaniswamy SK, Matthews N, Molina C, Kurtz M, Grotewold E: AGRIS: Arabidopsis gene regulatory information server, an information resource of Arabidopsis cis-regulatory elements and transcription factors. BMC Bioinformatics 2003, 4:25.

79. Yilmaz A, Mejia-Guerra MK, Kurz K, Liang X, Welch L, Grotewold E: AGRIS: the Arabidopsis Gene Regulatory Information Server, an update. Nucleic Acids Res 2011, 39(Database issue):D1118-D1122.

80. Yoo S-D, Cho Y-H, Sheen J: Arabidopsis mesophyll protoplasts: a versatile cell system for transient gene expression analysis. Nat Protoc 2007, 2(7):1565-1572.

doi:10.1186/1471-2229-12-179

Cite this article as: Stamm et al:: Insights into the molecular mechanism of RGL2-mediated inhibition of seed germination in Arabidopsis thaliana. BMC Plant Biology 2012 12:179.

\section{Submit your next manuscript to BioMed Central and take full advantage of:}

- Convenient online submission

- Thorough peer review

- No space constraints or color figure charges

- Immediate publication on acceptance

- Inclusion in PubMed, CAS, Scopus and Google Scholar

- Research which is freely available for redistribution 